\title{
Novel metasurface phase-modulation mechanism
}

\author{
Qinghua Song (iD ${ }^{1 凶}$
}

\section{Science, 373(6559), 1133-1137, (2021)}

https://doi.org/10.1126/science.abj3179

The past few years have witnessed exciting developments in non-Hermitian physics, showing unconventional phenomena and unique features associated with exceptional points (EPs). EPs exist in many open systems, leading to a spectral singularity. The research team from CNRS-CRHEA in France collaborating with the University of California, Berkeley in US utilizes the topological feature around an EP to introduce a novel design in metasurface to achieve a new wavefront phase encoding technique. They show that the intriguing polarization response of singular plasmonic meta-atoms encircling an $E P$ leads to $2 \pi$-phase modulation on a chosen outgoing channel, which is topologically protected by the EP. In auxiliary, combining the exceptional topological phase with Pancharatnam-Berry phase, they achieve arbitrary wavefront engineering on cross polarization channels independently. Their breakthrough explorations not only provide a new degree of freedom to address optical phase in a full $2 \pi$ range, but also open the way to a new class of optical and photonic applications.

\section{Conflict of interest}

The author declares no competing interests.

Received: 25 August 2021 Accepted: 25 August 2021

Published online: 14 September 2021 